\title{
Thyroid tumor cells-fibroblasts crosstalk: role of extracellular vesicles
}

\author{
Rocío del Carmen Bravo-Miana ${ }^{1,2}$, Ana Belén Della Vedova ${ }^{1,2}$, Ana Lucía De Paul ${ }^{3,4}$, María Mónica Remedi',2, \\ María Laura Guantay ${ }^{1,2}$, Mónica Beatriz Gilardoni ${ }^{1,2}$, Claudia Gabriela Pellizas ${ }^{1,2}$ and Ana Carolina Donadio ${ }^{1,2}$ \\ ${ }^{1}$ Universidad Nacional de Córdoba, Facultad de Ciencias Químicas, Departamento de Bioquímica Clínica, Córdoba, Argentina \\ ${ }^{2}$ Centro de Investigaciones en Bioquímica Clínica e Inmunología (CIBICl-CONICET), Haya de la Torre y Medina Allende, Ciudad Universitaria, Córdoba \\ (X5000HUA), Argentina \\ 3Universidad Nacional de Córdoba, Facultad de Ciencias Médicas, Centro de Microscopía Electrónica, Córdoba, Argentina \\ ${ }^{4}$ Instituto de Investigaciones en Ciencias de la Salud (INICSA-CONICET), Av. Enrique Barros y Enfermera Gordillo, Ciudad Universitaria, Córdoba, Argentina
}

Correspondence should be addressed to C G Pellizas or A C Donadio: claudia@fcq.unc.edu.ar or anacarol@fcq.unc.edu.ar

C G Pellizas is a member of the editorial board of Endocrine Connections. She was not involved in the editorial or review process for this paper, on which she is listed as an author

\begin{abstract}
Tumor-stroma crosstalk leads to a tumor-promoting microenvironment. In this milieu, extracellular vesicles (EVs) are protagonists in cell-cell communication. Despite thyroid cancer being the most common endocrine malignancy, the contribution of the tumor microenvironment to thyroid cancer progression is still largely underexplored. We focused on the role of thyroid tumor cell-fibroblast interaction and EVs as mediators of tumorstroma interplay, in the promotion of thyroid tumor aggressiveness. Thyroid tumor (TPC-1, 8505c) or non-tumor thyroid cells (NThyOri) were co-cultured with human fibroblasts (Fb). Thyroid cell migration was investigated by the wound-healing assay and actin-network staining. Cell-CD147 expression was characterized by flow cytometry. EVs, obtained by ultracentrifugation of conditioned media (CMs), were characterized by transmission electron-microscopy and CD81 and CD147 expression. Metalloproteinases (MMPs) were evaluated by zymography in CMs. A migratory phenotype was triggered in thyroid tumor cells treated with $\mathrm{CMs}$ from $\mathrm{Fb}$ or from Fb-thyroid tumor cell co-cultures. Fb-thyroid cell co-cultures induced the secretion of proMMP9 and proMMP2 and led to a significant MMP2 activation in CMs. Fb, thyroid cells and Fb-thyroid cell co-cultures released EVs, and remarkably, EVs released by Fb-thyroid tumor cell co-cultures induced the secretion of proMMP2 and the expression of MMP2 from normal Fb. A significant CD147 expression was demonstrated in Fb-thyroid tumor cell-derived EVs. These findings reveal the role of $\mathrm{Fb}$ and thyroid tumor cell-Fb interaction in the promotion of a microenvironment suitable for thyroid tumor progression. Moreover, they highlight, for the first time, the role of thyroid tumor cell-Fb interaction in the production of specialized EVs.
\end{abstract}

\section{Key Words}

- extracellular vesicles

- fibroblasts

- matrix metalloproteinases

- thyroid

- tumor microenvironment

\section{Introduction}

Carcinomas comprise tumor and non-tumor interacting cells. In this context, tumor and stroma cell interaction gives rise to several signals that affect stromal cell behavior, leading to a tumor-promoting microenvironment $(1,2)$. A prominent functional role for fibroblasts $(\mathrm{Fb})$ in cancer progression and metastasis has been demonstrated in many studies (3), with the ability of cancer cells to become migratory and invade into surrounding tissues and distant organs being another crucial step in cancer development. In these milieus, metalloproteinases (MMPs) play a
Endocrine Connections (2020) 9, 506-518 
relevant role by participating in the extracellular matrix (ECM) turnover and altering the cell-ECM and cell-cell interactions, causing tissue architecture disturbances that enhance tumor growth and aggressiveness $(4,5)$.

CD147 is a transmembrane glycoprotein involved in the secretion and activation of MMPs (6). It is highly expressed in many malignant tumor cells (7) and has been related to the tumor-stroma interaction $(8,9)$. In this regard, we reported increased CD147 and MMP9 expression in metastasized liver samples from tumorbearing rats (10), with these results being reproduced and blocked in vitro using anti-CD147 antibodies in co-cultures of tumor and normal rat liver cells, thus highlighting the role of CD147 in mediating tumor-host interactions.

Tumor-stroma interplay involves the exchange of cellular information. Although cell-cell interactions and the secretion of effector molecules are well-known mediators of intercellular crosstalk, recent research has identified extracellular vesicles (EVs) as being another protagonist in cell-cell communication $(11,12)$. EVs are heterogeneous populations of nano- to micro-sized particles released through the endosomal pathway or by budding from the plasma membrane (12) and are vehicles for the horizontal transfer of proteins, nucleic acids and other metabolites to neighboring cells or to distant anatomic sites. Tumor-derived EVs are able to alter the phenotype of recipient cells, transform benign cells and depress the immune response, induce epithelialmesenchymal transition and support endothelial proliferation and blood vessel sprouting $(13,14)$. Interestingly, CD147 has been described in EVs derived from multiple myeloma and breast cancer cell lines, as well as in EVs from plasma samples of multiple myeloma, metastatic breast cancer, colorectal carcinoma and other epithelial neoplasia patients $(15,16,17)$.

Thyroid cancer is the most common malignancy of the endocrine system, with an increasing incidence rate recorded over the last three decades (18). The contribution of the tumor microenvironment to the development of thyroid cancer is beginning to be better understood. With respect to this, in thyroid neoplasia, the Fb recruitment and ECM remodelling have been reported as pivotal features of the tumor milieu, such as in promoting thyroid cancer progression in a mouse model of papillary thyroid carcinoma (PTC) (19). Previously, using a rat tumor model, Saitoh and coworkers (20) demonstrated that $\mathrm{Fb}$ confer growth-promoting advantages to thyroid carcinoma cells, both in vivo and in vitro. Interestingly, this growthpromoting activity was mediated by soluble factors present in the Fb-conditioned medium. Noteworthy, we have recently reported that soluble factors present in the CMs of human activated $\mathrm{Fb}$ promoted the proliferation and invasion of the follicular thyroid cancer cell line, FTC-133 (21). Although there have been proposed roles for $\mathrm{Fb}$ in thyroid tumor progression, the relevance of thyroid tumor cell-Fb crosstalk in thyroid tumor promotion still remains underexplored. Thus, using a simulation of the thyroid tumor microenvironment, we analyzed in a human model the role of Fb-thyroid tumor cell interaction in the promotion of tumor aggressiveness, with emphasis on the description of EV-mediating tumor-stroma interplay.

\section{Materials and methods}

\section{Cell lines and cell maintenance}

The human papillary thyroid cancer cell line (TPC-1) used was kindly provided by Dr Guillermo Juvenal (Comisión de Energía Atómica, Buenos Aires, Argentina), and the anaplastic thyroid cancer cell (8505c) and the non-tumor thyroid (NThyOri) cell lines were kindly donated by Dr Pilar Santisteban (Instituto de Investigaciones Biomédicas Alberto Sols, Madrid, Spain). Normal human skin Fb were used as stromal representative cells. They were obtained from anonymized healthy volunteers, with the protocol being approved by the Ethical Committee of Hospital Nacional de Clínicas, Universidad Nacional de Córdoba. Cells were cultured in high glucose DMEM media (HG-DMEM; Sigma Aldrich) or RPMI 1640 media (Gibco; Life Technologies) supplemented with $10 \% \mathrm{v} / \mathrm{v}$ fetal bovine serum (FB) (Natocor, Córdoba, Argentina) and penicillin 100 units/mL-streptomycin $100 \mu \mathrm{g} / \mathrm{mL}$ (Gibco) in a humidified $5 \% \mathrm{CO}_{2}$ atmosphere at $37^{\circ} \mathrm{C}$.

In this study, cell migration was characterized by the rhodamine-conjugated phalloidin staining of cellfilopodia and lamellipodia, as well as by the in vitro wound-healing assay. The release of EVs was studied by transmission electron microscopy (TEM) and Western blotting assays. The effect of cell-cell and EVs-cell interaction in the proteolytic activity of MMPs was evaluated by zymography.

\section{Co-cultures of $\mathrm{Fb}$, thyroid tumor and non-tumor cells}

Fb (600,000 cells) and human thyroid tumor (TPC-1 or 8505c) or non-tumor (NThyOri) cells $(600,000$ cells) were mixed and plated together in 100-mm-diameter plates (contact-dependent co-cultures). The expressions 
'Fb-TPC-1, Fb-8505c and Fb-NThyOri' are used throughout this work to indicate the co-culture of $\mathrm{Fb}$ and any of the indicated thyroid cells. Fb, TPC-1, 8505c and NThyOri isolated cells were seeded as controls, using the same number of cells as in the corresponding co-cultures (22). The cells were first cultured in serum-supplemented medium for $72 \mathrm{~h}$. Then, the monolayers were washed with PBS and the culture medium was changed to serumfree medium (SFM, $9 \mathrm{~mL} /$ plate) for $48 \mathrm{~h}$.

The supernatants were collected and immediately cooled to $4^{\circ} \mathrm{C}$, with cells and cellular debris being removed by sequential centrifugation at $300 \boldsymbol{g}$ for $10 \mathrm{~min}$, followed by $3000 \boldsymbol{g}$ for $20 \mathrm{~min}$. Finally, the CMs were stored at $-80^{\circ} \mathrm{C}$ for zymographic and cell migration studies.

\section{Actin immunofluorescence}

Thyroid cells were grown to 75\% confluence on uncoated glass coverslips in HG-DMEM-FB 10\% (TPC-1, 8505c) or RPMI-FB 10\% (NThyOri) in 24-well plates. The monolayers were then stimulated for $24 \mathrm{~h}$ at $37^{\circ} \mathrm{C}$ with $\mathrm{CMs}$ obtained from isolated Fb, TPC-1, 8505c or NThyOri cells and from Fb-TPC-1, Fb-8505c and Fb-NThyOri co-cultured cells, diluted 1:1 with fresh culture medium-FB $2 \%$. After incubation, coverslips were fixed for $20 \mathrm{~min}$ at room temperature in $4 \% \mathrm{w} / \mathrm{v}$ paraformaldehyde- $4 \% \mathrm{w} / \mathrm{v}$ sucrose diluted in PBS, before being permeabilized with PBS- $0.2 \%$ v/v Triton-X100 and stained with rhodamine-conjugated phalloidin (Invitrogen, Life Technologies Corporation). Finally, slides were incubated with the nuclear stain DAPI, mounted with FluorSave-Reagent (Calbiochem) and examined with a Nikon ECLIPSE TE2000-U inverted microscope. The presence of lamellipodia and filopodia on the cells was considered to be a signal of migratory phenotype. The evaluation and quantification of cells with these characteristics was carried out in ten randomly selected fields (63X). Control cultures were performed using fresh culture medium-FB 1\%.

\section{In vitro wound healing assay}

TPC-1, 8505c and NThyOri cells were plated in 24-well plates in HG-DMEM- or RPMI-FB 10\% and grown to confluence. After thyroid cell monolayers were treated with $75 \mu \mathrm{g} / \mathrm{mL}$ mitomycin C, a scratch was made in each confluent monolayer using a sterile $200 \mu \mathrm{L}$ pipette tip. Cell monolayers were rinsed twice with warm PBS and finally incubated with SFM or CMs from isolated and co-cultured thyroid cells in a 1:1 mixture with SFM. Immediately after wounding $(0 \mathrm{~h})$ and after $6-24 \mathrm{~h}$ of incubation at $37^{\circ} \mathrm{C}$, phase-contrast images of each scratch were obtained digitally using a Nikon ECLIPSE TE2000-U inverted microscope. The distance between the edges of the wounds was measured at three points in each picture on the same field of interest. The percentage of the remaining distance between edges was calculated using the width of the scratch at each indicated time point divided by that recorded at the beginning of the assay $\times 100$. Each result was the mean of three independent experiments.

\section{CD147 expression in Fb and thyroid co-cultured cells}

CD147 expression was analyzed in Fb, TPC-1, 8505c and NThyOri isolated cells and in Fb-TPC-1, Fb-8505c and Fb-NThyOri co-cultured cells by flow cytometry. For Fb identification in Fb-thyroid cell co-cultures, Fb were stained with Cell Proliferation Dye eFluor 670 (CPD, eBioscience) following the manufacturer's instructions. CPD-positive $\mathrm{Fb}$ were cultured with TPC-1, 8505c and NThyOri cells (as stated above) and isolated CPDpositive Fb, TPC-1, 8505c and NThyOri cultures were used as controls. After culture, the cells were collected and the CD147 expression in isolated and co-cultured Fb and thyroid cells was evaluated using the CD147-FITC (BD Pharmingen, BD Biosciences) antibody, following the manufacturer's instructions. Data acquisition was performed with a FACS Canto II flow cytometer (BD Biosciences), using FlowJo software for the final analysis. The percentages of CD147(+) CPD-positive $\mathrm{Fb}$ and CD147(+) CPD-negative thyroid cells were calculated in isolated and co-cultured cells, with each result being the mean of four independent experiments.

\section{Isolation of extracellular vesicles}

EV isolation was performed by serial centrifugation, as described by Desrochers and coworkers (23) with slight modifications. Briefly, culture supernatants, derived from the same number of final cells after culture (24), were removed from the cells and centrifuged at $300 \boldsymbol{g}$ at $4^{\circ} \mathrm{C}$ for $10 \mathrm{~min}$ and then again at $3000 \boldsymbol{g}$ at $4^{\circ} \mathrm{C}$ for $20 \mathrm{~min}$ to remove intact cells and cell debris, respectively. To isolate the EV-enriched populations, the CMs obtained as described previously were then ultracentrifuged at $100,000 \boldsymbol{g}$ at $4^{\circ} \mathrm{C}$ for $90 \mathrm{~min}$ (100K-EVs).

For the $\mathrm{Fb}$ stimulation assays, the 100K-EVs were resuspended in $2.0 \mathrm{~mL}$ of SFM and added to $\mathrm{Fb}(200,000$ cells). As a control for all experiments involving the EVs, SFM was added to another set of recipient cells.

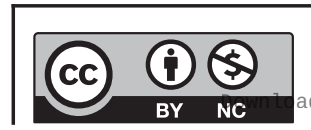

This work is licensed under a Creative Commons Attribution-NonCommercial 4.0 International License. ded from Bioscientifica.com at 04/26/2023 11:07:08AM 
For the Western blot assays, the pelleted 100K-EVs were lysed with sample buffer $2 \mathrm{X}$.

\section{Transmission electron microscopy}

The ultracentrifuged EVs were resuspended in $4 \% \mathrm{w} / \mathrm{v}$ paraformaldehyde, and $5 \mu \mathrm{L}$ of EVs preparation were dropped on the formvar-carbon coated EM grids and postfixed in 1\% w/v glutaraldehyde. The grid was washed with distilled water, and then the samples were contrasted in $2 \% \mathrm{w} / \mathrm{v}$ uranyl oxalate ( $\mathrm{pH} 7$ ) followed by $2 \% \mathrm{w} / \mathrm{v}$ methyl cellulose for negative contrasting.

For CD147-immunogold labelling, fixed EVs were incubated with the anti-CD147 antibody (dilution 1:25; Affimetrix, eBioscience), and the immunoreactive sites were labeled with anti-mouse secondary antibody conjugated to $15-\mathrm{nm}$ colloidal gold particles (1:30; Electron Microscopy Sciences, Hatfield, PA, USA). The EVs were visualized using a Zeiss Leo 906-E electron microscope, and for cell ultrastructural characterization, isolated and co-cultured thyroid cells were fixed and processed as previously described (25).

\section{Fb stimulation assay}

Fb were seeded in six-well culture plates $(200,000 \mathrm{Fb} /$ well) in HG-DMEM-FB 10\% until confluence. The Fb monolayers were then washed with warm PBS and stimulated for $24 \mathrm{~h}$ with or without $100 \mathrm{~K}-\mathrm{EV}$ s, obtained from isolated or co-cultured thyroid cells and prepared as indicated previously (see 'Isolation of extracellular vesicles' section). After incubation, the culture supernatants were cleared of intact cells and cell debris and saved at $-80^{\circ} \mathrm{C}$ for zymographic determination of MMP9 and MMP2 activity.

\section{Zymography}

Gelatinolytic activity in the CMs $(30 \mu \mathrm{L})$ was demonstrated by zymography using gelatin as a substrate as was previously described (10). Enzyme activity was visualized as a colorless band on a black background.

\section{Western blot assay}

The $100 \mathrm{~K}-\mathrm{EV}$ s pellets were suspended in $60 \mu \mathrm{L}$ of $2 \mathrm{X}$ sample buffer on ice, vortexed every $5 \mathrm{~min}$ for $20 \mathrm{~min}$ and boiled at $95^{\circ} \mathrm{C}$ for $5 \mathrm{~min}$. Samples were separated by sodium dodecyl sulfate-PAGE (12\% SDS-PAGE) and electrotransferred to nitrocellulose membranes (BioRad). Nonspecific binding sites were blocked for $1 \mathrm{~h}$ at room temperature in Tris-buffered saline containing 5\% w/v non-fat dry milk and $0.1 \% \mathrm{v} / \mathrm{v}$ Tween 20 . The blots were incubated overnight at $4^{\circ} \mathrm{C}$ with primary antibodies against human CD147 (dilution 1:500; Affimetrix) and CD81 (dilution 1:200; Santa Cruz Biotechnology) and then incubated for $2 \mathrm{~h}$ at room temperature with appropriate secondary IR Dye-tagged antibodies, after which they were visualized using an Odyssey IR imager (LI-COR Biosciences).

\section{Statistical analysis}

Values were expressed as mean \pm s.E.M. The statistical significance of differences among means was determined by a parametric or non-parametric ANOVA test and unpaired $t$-test. $P<0.05$ was considered to indicate a statistically significant difference.

\section{Results}

\section{Soluble factors of Fb or Fb-thyroid tumor cell CMs induce a migratory phenotype in thyroid tumor cells}

Concerning cell migration, dynamic actin structures such as stress fibers, filopodia and lamellipodia are arranged by the cells to be able to sense and move in their environment (26). To analyze the effect of CMs in the organization of filopodia, lamellipodia and stress-fibers in tumor and non-tumor thyroid cells, we obtained immunofluorescent images of rhodamine-conjugated phalloidin stained TPC-1, 8505c and NThyOri cells after their treatment with medium or CMs from Fb, TPC-1, 8505c, NThyOri cells or from Fb-TPC-1, Fb-8505c and Fb-NThyOri co-cultured cells. We recorded the number of cells exhibiting either lamellipodia or filopodia as an indirect measure of the migratory phenotype of thyroid cells (Fig. 1). The overall change in the number of cells with lamellipodia and/or filopodia followed a similar pattern in TPC-1 and 8505c cells, with a significant increase in the observed percentage of TPC-1 and 8505c cells with lamellipodia and/or filopodia after their incubation with $\mathrm{Fb} \mathrm{CMs}$, with respect to the control condition (medium). In turn, a significantly higher proportion of lamellipodia and filopodia positive8505c cells was also observed with the addition of Fb-8505c CMs. A similar trend in the percentage of TPC-1 cells with lamellipodia and/or filopodia was seen following TPC-1 treatment with Fb-TPC-1 CMs, although no significant differences were found vs control conditions. No changes were observed in the migratory characteristics

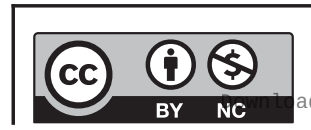

This work is licensed under a Creative Commons Attribution-NonCommercial 4.0 International License. ded from Bioscientifica.com at 04/26/2023 11:07:08AM 

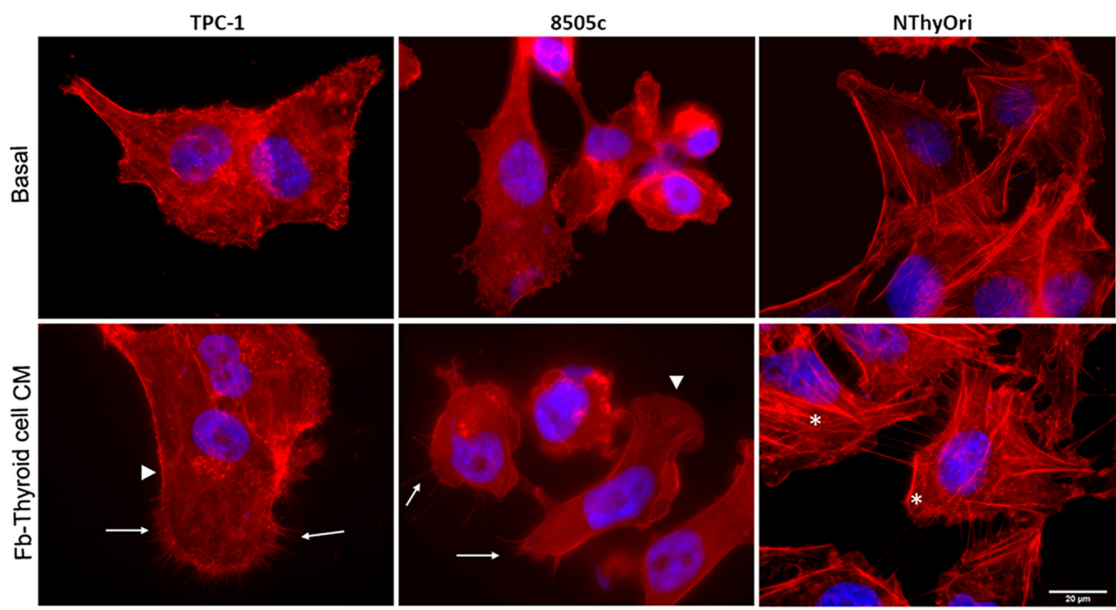

Figure 1

$\mathrm{Fb}$ and $\mathrm{Fb}$-thyroid tumor cell CMs promote filopodia and lamellipodia formation in thyroid tumor cells. Representative images of TPC-1, $8505 \mathrm{c}$ and NThyOri cells incubated for $24 \mathrm{~h}$ with medium (Basal) and Fb-TPC-1, Fb-8505c and Fb-NThyOri CMs, respectively (Fb-Thyroid cell CM) and then stained with rodhamine-conjugated phalloidin and visualized at 100X using a Nikon ECLIPSE TE2000-U inverted microscope. Filopodia are indicated by the white arrows, lamellipodia by the white arrowheads and stress fibers by the asterisks. Bar: $20 \mu \mathrm{m}$.

of NThyOri cells incubated with medium, Fb, NThyOri or Fb-NThyOri CMs (Table 1).

\section{Soluble factors of $\mathrm{Fb}$ and $\mathrm{Fb}-8505 \mathrm{C}$ CMs promote 2D-migration of $8505 \mathrm{c}$ tumor cells}

To further investigate the effect of the CMs from Fb-thyroid cell co-culture on thyroid cell migration, we examined the ability of TPC-1 (Fig. 2A), 8505c (Fig. 2B) and NThyOri (Fig. 2C) cells to migrate from a confluent monolayer into an area devoid of cells at 6 and $24 \mathrm{~h}$. Each thyroid cell monolayer was stimulated with basal medium, Fb CMs, isolated thyroid cell CMs or Fb-thyroid cell CMs. Results show that the treatment of $8505 \mathrm{c}$ with both CMs for $24 \mathrm{~h}$, those from $\mathrm{Fb}$ and $\mathrm{Fb}-8505 \mathrm{c}$ co-cultures, resulted in cells repopulating the cell-free area at a higher rate (Fig. $2 \mathrm{~B}$ and $\mathrm{D} ; 26.3 \pm 2.0 \%$ and $27.6 \pm 0.4 \%$ remnant cell-free area; $P<0.01$ and $P<0.05$ respectively) compared with

Table 1 Percentage of filopodia and/or lamellipodia-positive TPC-1, 8505c and NThyOri cells after the indicated treatments.

\begin{tabular}{|c|c|c|c|}
\hline Cells & TPC-1 & $8505 c$ & NThyOri \\
\hline Treatment & $\%$ & $\%$ & $\%$ \\
\hline Medium & 35 & 19 & 9 \\
\hline $\mathrm{Fb}$ CMs & $78^{a}$ & $52^{a}$ & 17 \\
\hline TPC-1 CMs & 33 & & \\
\hline Fb-TPC-1 CMs & 61 & & \\
\hline 8505c CMs & & 22 & \\
\hline Fb-8505c CMs & & $60^{b}$ & \\
\hline NThyOri CMs & & & 12 \\
\hline Fb-NThyOri CMs & & & 18 \\
\hline
\end{tabular}

The number of cells with lamellipodia and/or filopodia were counted in ten $(63 \mathrm{X})$ randomly selected fields. $P$-values $<0.05$ (Fisher Exact test) are indicated as a for TPC -1 and 8505 c cells treated with Fb CMs vs basal condition (Medium) and as ${ }^{\mathrm{b}}$ for 8505 c cells treated with Fb-8505c CMs vs basal condition.

CMs, Conditioned media. 8505c cell migration in the basal medium. However, TPC-1 and NThyOri cell migration did not reveal any significant changes for any of the assayed conditions vs control migration (medium) (Fig. 2A and $\mathrm{C}$ respectively). In addition, no changes in cell migration were registered following $6 \mathrm{~h}$ of incubation.

To note, cell movements dependent on matrix degradation could not be revealed by the wound healing assay.

\section{Fb, thyroid cells and Fb-thyroid cell co-cultures secrete EVs to CMs}

It is well-known that EVs provide a way to interchange information between homotypic and heterotypic cells in the tumor microenvironment (12). To address whether EVs could have been progressively released in our experimental system, we first used transmission electron microscopy (TEM) to analyze isolated Fb, TPC-1, 8505c and NThyOri cells as well as Fb-TPC- 1, Fb-8505c and Fb-NThyOri co-cultured cells for evidence of shedding activity. Figure 3 shows a representative image of an Fb-TPC-1 cell co-culture. The plasma membrane of TPC-1 cells co-cultured with $\mathrm{Fb}$ was rich in spherical and villi-like cell protrusions (Fig. 3A, insert B), with similar findings also being observed in isolated TPC-1, 8505c and NThyOri cells, as well as in $8505 \mathrm{c}$ and NThyOri cells co-cultured with $\mathrm{Fb}$ (data not shown). In striking contrast, normal Fb revealed a smoother cell surface (Fig. 3C). To verify if EVs were being released, we collected the CMs of isolated Fb, TPC-1, 8505c and NThyOri as well as of Fb-TPC-1, $\mathrm{Fb}-8505 \mathrm{c}$ and Fb-NThyOri co-cultures and processed them as described in the 'Materials and methods' section for TEM analysis. EVs could be detected in all the 100K-EVs fractions analyzed, with Fig. 3D, E, F and G showing representative images of 100K-EVs released 

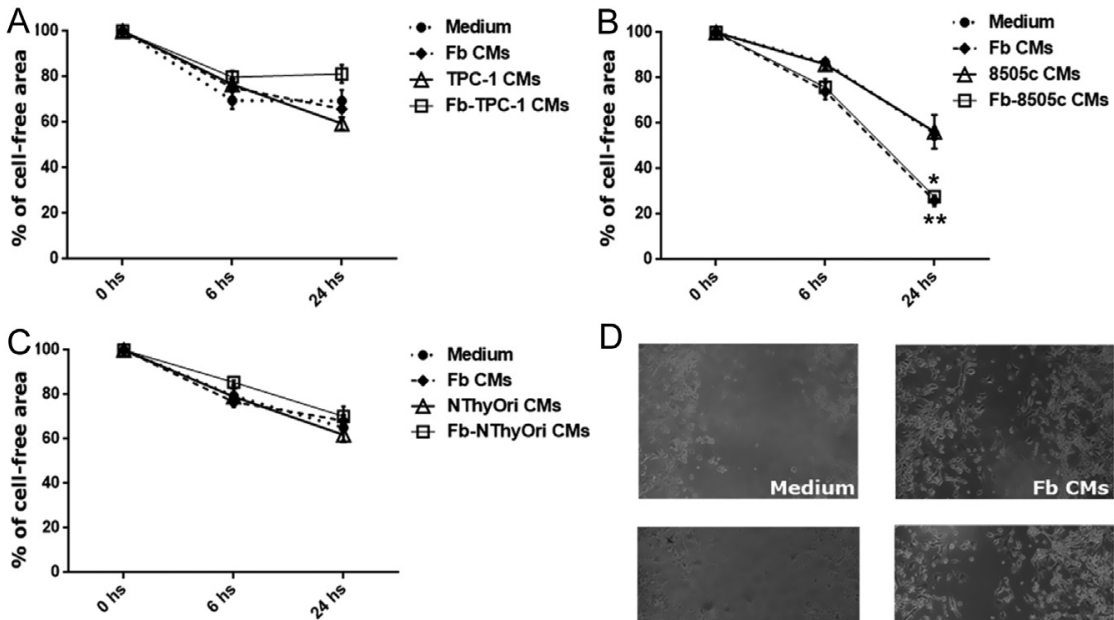

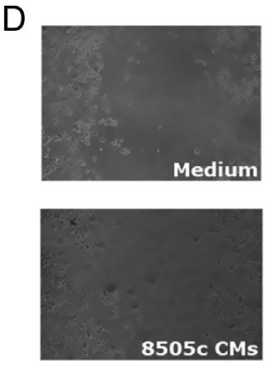

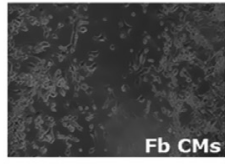

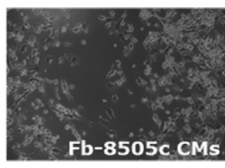

Figure 2

In vitro wound healing assay. The ability of TPC-1, $8505 \mathrm{C}$ and NThyOri cells to migrate into the cell-free wound area after the indicated treatments was assessed at 6 and $24 \mathrm{~h}$. The percentage of (A) TPC-1, (B) 8505c and (C) NthyOri cell migration was calculated as described in the Materials and Methods section. Data are presented as the mean value of three independent experiments (mean \pm s.E.M.). The asterisks $\left({ }^{*} P<0.01, * P<0.05\right.$, Kruskal-Wallis test, Dunn's post test) indicate a significant $8505 \mathrm{c}$ cell migration when comparing $\mathrm{Fb}-\mathrm{CM}$ and $\mathrm{Fb}-8505 \mathrm{c}$ CM treatments vs basal condition (Medium), respectively. (D) Representative microphotographs of $8505 \mathrm{c}$ cell-wounds incubated with medium, Fb, 8505c and Fb-8505c CMs. CMs, Conditioned media. from TPC-1 (Fig. 3D), Fb-8505c (Fig. 3E) and Fb-TPC-1 co-cultures (Fig. 3F and G), where the characteristic cupshaped morphology of EVs can be observed. Similar patterns were also obtained in isolated $8505 \mathrm{c}$, NThyOri and Fb-NthyOri cell co-cultures (data not shown).
When analyzed by TEM, the majority of vesicles pelleting at $100,000 \boldsymbol{g}$, obtained from $\mathrm{Fb}$, isolated and co-cultured thyroid cells, were of the size generally described for exosomes (i.e. $50-200 \mathrm{~nm}$ ), with about $10-20 \%$ of the remaining EVs being larger than $200 \mathrm{~nm}$ (Fig. 3H).
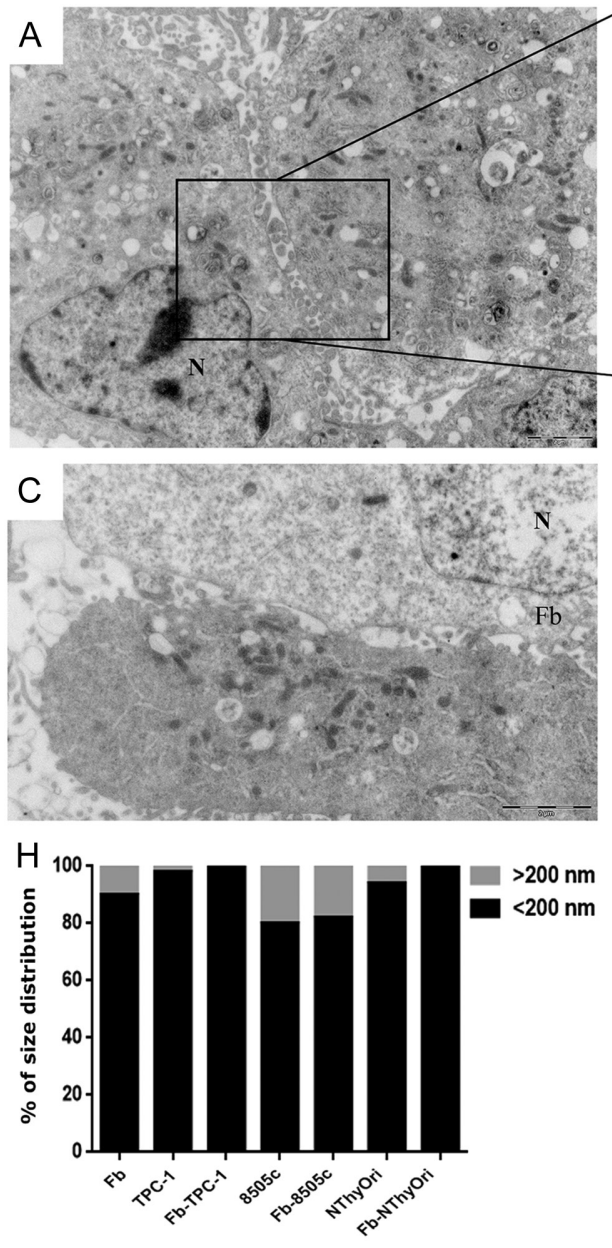
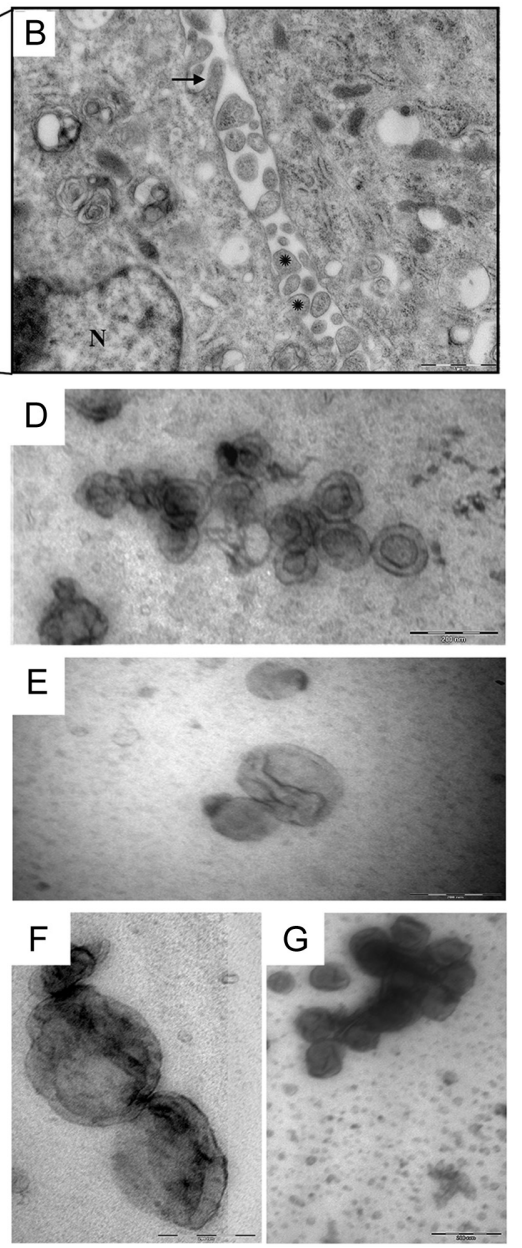

Figure 3

Representative transmission electron microphotograph of Fb-TPC-1 cell co-cultures and 100K-EVs. (A) Fb-TPC-1 co-cultured cells. Note the 'villi like' (B, arrow) and rounded ( $\mathrm{B}$, asterisks) membrane projections in TPC-1 cells and the smoother cell surface in $\mathrm{Fb}(\mathrm{C})$. (D, E, F and G) Representative transmission electron images of isolated and grouped $100 \mathrm{~K}-\mathrm{EV}$ s released from TPC-1 (D), Fb-8505c (E) and Fb-TPC-1 co-cultured cells $(F, G)$. Note the characteristic cup-shaped morphology of 100K-EVs. (H) Transmission electron microscopy analysis of size distribution of the 100K-EVs from Fb, TPC-1, 8505c, NThyOri and Fb-TPC-1, Fb-8505c and Fb-NThyOri

co-cultures. The diameter of membrane-enclosed structures was determined using Image software for two independent experiments. 100K-EVs, 100,000-g-ultracentrifuged extracellular vesicles. Bars: $2 \mu \mathrm{m}(\mathrm{A}, \mathrm{C}) ; 1 \mu \mathrm{m}$ (B); $200 \mathrm{~nm}(\mathrm{D}, \mathrm{E}, \mathrm{F}, \mathrm{G})$. https://ec.bioscientifica.com https://doi.org/10.1530/EC-20-0113 (c) 2020 The authors Published by Bioscientifica Ltd

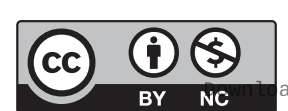

This work is licensed under a Creative Commons Attribution-NonCommercial 4.0 International License. ded from Bioscientifica.com at 04/26/2023 11:07:08AM 


\section{MMP2 activity increases in CMs from thyroid tumor cells co-cultured with Fb}

The gelatinolytic activity of MMP2 was measured in CMs obtained from cultures of Fb, TPC-1, 8505c and NThyOri, as well as in those from co-cultures of Fb-TPC-1, Fb-8505c and Fb-NThyOri cells. ProMMP2 and MMP2 activities were detected in the $\mathrm{Fb} \mathrm{CMs}$, while a very faint expression of proMMP2 could be detected in the CMs from all the thyroid cell lines tested. A significant increase in MMP2 activity was registered in CMs obtained from Fb-TPC-1 (Fig. 4B and C) and Fb-8505c (Fig. 4B and D) co-cultures compared with isolated thyroid tumor cells $(P<0.01$ and $P<0.05$, respectively). It is noteworthy that MMP2 activity was not detected in CMs from isolated NThyOri cells and was only slightly detected in Fb-NThyOri co-cultures (Fig. 4B and E). Moreover, there was a significantly higher prevalence of the active form of MMP2 in CMs obtained from Fb-TPC-1 and Fb-8505c co-cultures with respect to Fb-NThyOri CMs.
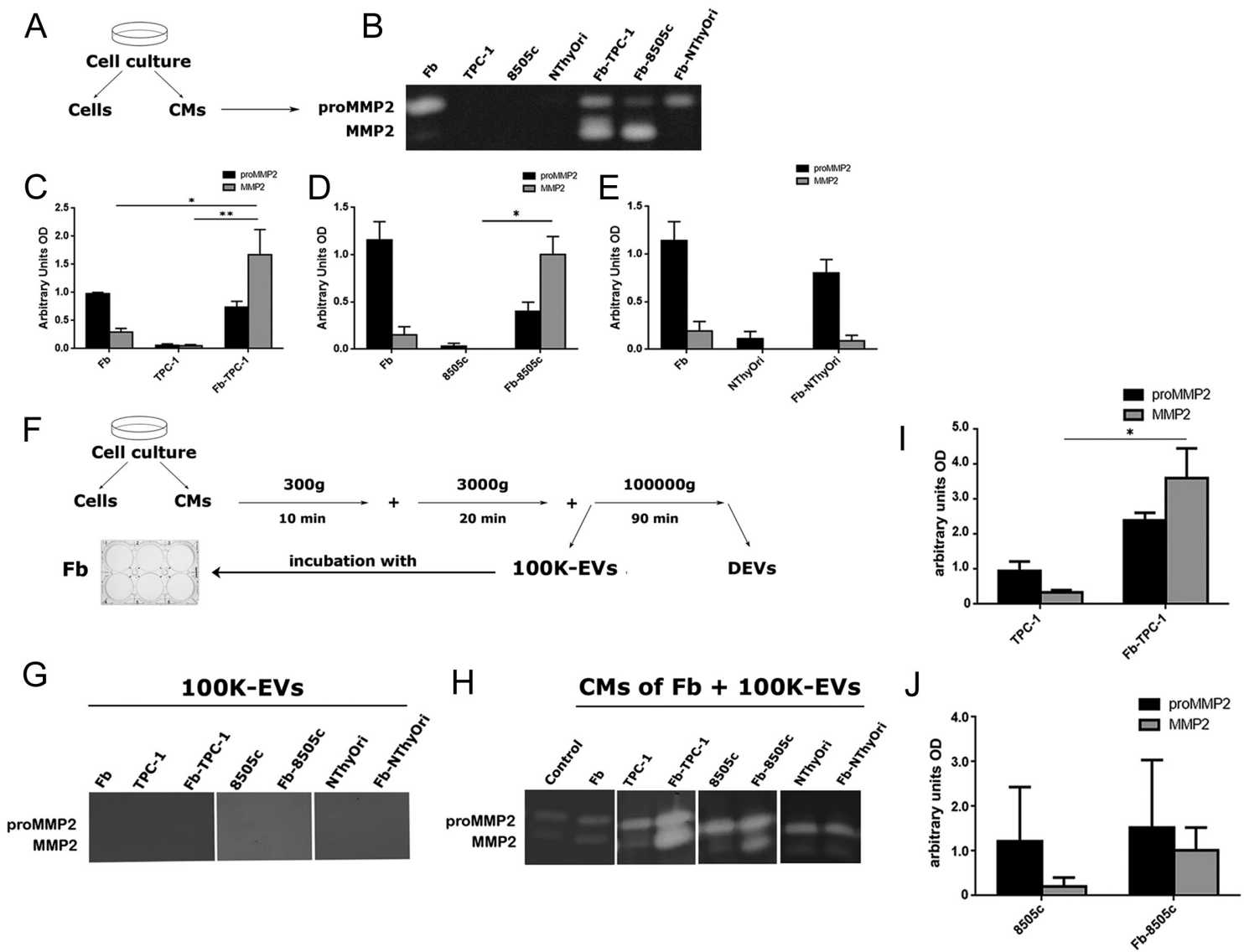

\section{Figure 4}

ProMMP2 and MMP2 activity in CMs from Fb, isolated or co-cultured thyroid cells and from Fb stimulated with 100K-EVs from isolated and co-cultured thyroid cells. (A) Schematic representation of $\mathrm{Fb}$, thyroid cell and Fb-thyroid cell culture and CM study. (B) Representative zymogram showing proMMP2 and MMP2 gelatinolytic activity in Fb, TPC-1, 8505c and NthyOri and Fb-TPC-1, Fb-8505c and Fb-NThyOri co-cultured cells. Areas of protease activity are indicated by clear bands in the gel for proMMP2 and MMP2. (C, D, E) Densitometric analysis of proMMP2 and MMP2 in CMs from (C) Fb, TPC-1 and Fb-TPC-1 co-cultured cells; (D) Fb, 8505c and Fb-8505c co-cultured cells and (E) Fb, NThyOri and Fb-NThyOri co-cultured cells. Results are expressed as the mean \pm S.E.M. of at least four independent determinations. A significant increase was observed in MMP2 activity in CMs from co-cultured Fb-TPC-1 vs TPC-1 and $\mathrm{Fb}$ ( $* * P<0.01$ and $* P<0.05$, respectively) and in Fb-8505c cells compared to $8505 \mathrm{c}$ cells ( $* P<0.05$; Kruskal-Wallis test, Dunn's post test). ( $\mathrm{F}$ ) Schematic representation of 100K-EVs obtentions and Fb $+100 \mathrm{~K}-\mathrm{EV}$ s CMs preparation. (G) Representative zymograms of 100K-EVs from Fb, TPC-1, 8505c, NThyOri and Fb-TPC-1, Fb-8505c and Fb-NThyOri co-cultured cells, with no gelatinolytic activity being detected. (H) Representative zymograms showing proMMP2 and MMP2 gelatinolytic activity in Fb-CM upon stimulation with medium (control) or 100K-EVs (CMs of Fb + 100K-EVs) from Fb, TPC-1, 8505c, NThyOri and Fb-TPC-1, Fb-8505c and Fb-NThyOri co-cultured cells. Areas of protease activity are indicated by clear bands in the gel. (I, J) Densitometric analysis of proMMP2 and MMP2 activity in Fb-CMs. A significant increase in MMP2 activity was observed in Fb-CMs upon stimulation with $100 \mathrm{~K}$-EVs from Fb-TPC-1 cells vs $100 \mathrm{~K}-$ EVs from TPC- 1 cells ( $* P<0.05$. Kruskal-Wallis test, Dunn's post test). () A trend to a higher MMP2 activity was observed in Fb-CMs stimulated with $100 \mathrm{~K}-\mathrm{EV}$ s from Fb-8505c vs $8505 \mathrm{c}$ cells. No changes in MMP2 activity were detected using $100 \mathrm{~K}$-EVs from NThyOri and Fb-NThyOri co-cultured cells ( $\mathrm{H}$, and data not shown). CMs, conditioned media; 100K-EVs, 100,000-g-ultracentrifuged extracellular vesicles; CMs of Fb + 100K-EVs, conditioned media from Fb upon stimulation with $100 \mathrm{~K} \mathrm{EVs;} \mathrm{DEVs,} \mathrm{depleted-EVs} \mathrm{media.}$

https://ec.bioscientifica.com https://doi.org/10.1530/EC-20-0113 (c) 2020 The authors Published by Bioscientifica Ltd

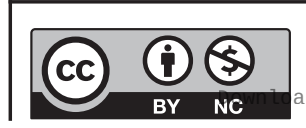

This work is licensed under a Creative Commons Attribution-NonCommercial 4.0 International License. ded from Bioscientifica.com at 04/26/2023 11:07:08AM 
Since previous results have shown proMMP2 and MMP2 activity in CMs from isolated and co-cultured cells and considering that MMPs have been described in EVs (27), we next evaluated MMP2 expression in EV-enriched fractions obtained from CMs of thyroid and Fb-thyroid cells co-cultures. Although no detectable MMP2 activity was observed in 100K-EVs under these experimental conditions (Fig. 4G), proMMP2 and MMP2 activities were detected in EV-depleted CMs (DEVs), which followed a similar expression pattern as the whole CMs previously tested (Supplementary Fig. 1, see section on supplementary materials given at the end of this article).

\section{EVs isolated from Fb-thyroid tumor cell CMs induce MMP2 activity in Fb}

EVs are key players in cell to cell communication, driving the production of a reactive tumor microenvironment (28). To evaluate the role of EVs in the expression of the MMPs previously described, we assayed the ability of EV-enriched fractions to induce the secretion of MMPs in Fb. To this aim, Fb were stimulated with $100 \mathrm{~K}-\mathrm{EVs}(\mathrm{Fb}+100 \mathrm{~K}-\mathrm{EVs})$ obtained from isolated Fb, TPC-1, 8505c and NThyOri as well as from Fb-thyroid cell co-cultures (Fb-TPC-1, Fb-8505c and Fb-NThyOri), with it being observed that proMMP2 and MMP2 enzymes were secreted to Fb-culture supernatants ( $\mathrm{Fb} \mathrm{CMs}$ ) upon $\mathrm{Fb}$ stimulation with 100K-EVs from isolated or Fb-co-cultured thyroid cells (Fig. 4H). It is noteworthy that a significant increase in active MMP2 was detected in $\mathrm{Fb}$ CMs upon Fb stimulation with 100K-EVs from Fb-TPC-1 compared with 100K-EVs from isolated TPC- 1 cells (Fig. $4 \mathrm{H}$ and I; $P<0.05$ ). A similar trend was also observed in $\mathrm{Fb} \mathrm{CMs}$ upon stimulation with $100 \mathrm{~K}-\mathrm{EV}$ s from $\mathrm{Fb}-8505 \mathrm{c}$ vs 100K-EVs from 8505c, although this did not reach statistical significance (Fig. $4 \mathrm{H}$ and J). Interestingly, no differences in the proMMP2 and MMP2 activities were registered when $\mathrm{Fb}$ were stimulated with 100K-EVs from Fb, Fb-NThyOri or NThyOri cells (Fig. 4H).

\section{proMMP9 activity increases in $\mathrm{Fb}-8505 \mathrm{C}-\mathrm{CMs}$ and in CMs of Fb stimulated with 100K-EVs from Fb-8505c co-cultures}

Gelatinolytic activity of MMP9 was evaluated in CMs from isolated and co-cultured cells, with only proMMP9 activity being detected in culture supernatants (Supplementary Fig. 2). Regarding proMMP9 expression, a significant increase in proMMP9 gelatinolytic activity was observed in Fb-8505c co-cultures related to isolated
Fb and 8505c cells. A trend toward a higher proMMP9 activity was also observed in Fb-TPC- 1 co-cultures vs isolated TPC-1 cells. As in the case of MMP2, proMMP9 was not detected in CMs from isolated NThyOri cells and was only slightly expressed in CMs from Fb-NThyOri co-cultures (Supplementary Fig. 2B and C).

We next evaluated MMP9 expression in EV-enriched fractions from isolated or co-cultured cells, but no detectable proMMP9 or MMP9 activity could be demonstrated (data not shown). However, upon stimulation of $\mathrm{Fb}$ with EV-enriched fractions, a trend to higher proMMP9 activity was detected in $\mathrm{CMs}$ of $\mathrm{Fb}$ stimulated with $100 \mathrm{~K}-\mathrm{EV}$ s from $\mathrm{Fb}-8505 \mathrm{c}$ vs $100 \mathrm{~K}-\mathrm{EV}$ s from 8505c cells (Supplementary Fig. 2E and F; $P=0.09$ ), although no significant changes could be demonstrated upon $\mathrm{Fb}$ stimulation with 100K-EVs from Fb-TPC-1 compared with $100 \mathrm{~K}-\mathrm{EV}$ s from isolated TPC-1. Finally, no changes in the expression of proMMP9 were registered in $\mathrm{Fb}$ CMs upon Fb stimulation with 100K-EVs from Fb or isolated or co-cultured NThyOri cells (Supplementary Fig. 2E and F).

\section{CD147 expression in Fb, thyroid cells and EVs from thyroid cells and Fb-thyroid cell co-cultures}

CD147 is expressed at high levels on the surfaces of various malignant cells, including thyroid carcinoma cells $(7,29)$. We evaluated the membrane expression of CD147 in isolated Fb, TPC-1, 8505c and NThyOri cells as well as in interacting Fb-TPC-1, Fb-8505c and Fb-NThyOri cells. The flow cytometric analysis revealed a high percentage of CD147 positive TPC-1 (78.4 \pm 4.6$)$, 8505c $(89.8 \pm 1.4)$ and NThyOri cells $(79.1 \pm 6.7)$, which did not change after TPC-1, 8505c or NThyOri co-culture with Fb $(79.7 \pm 3.8 ; 91.0 \pm 2.2$ and $77.4 \pm 8.4$, respectively, Fig. $5 \mathrm{~A})$. In addition, we tested $\mathrm{CD} 147$ expression in $\mathrm{Fb}$ and in Fb-TPC-1, Fb-8505c and Fb-NThyOri co-cultures, and similarly, we found that the percentage of CD147 positive $\mathrm{Fb}(42.6 \pm 11.1)$ did not alter significantly $(56.7 \pm 6.8$; $66.8 \pm 10.5 ; 72.6 \pm 3.2 ; P=0.12$, Fig. $5 \mathrm{~B}$ ) after Fb co-culture with TPC-1 (Fb-TPC-1), 8505c (Fb-8505c) or NThyOri cells (Fb-NThyOri), respectively. Even though CD147 was originally identified on the cell surface, CD147 could also be detected in EVs released from the cell $(8,9,16$, 30). We next explored CD147 expression in 100K-EV enriched fractions, with CD147 expression being detected by immunoelectron-cytochemistry in EVs obtained from both isolated thyroid and co-cultured cells, with Fig. 5C displaying a representative picture of CD147 expression in released EVs from Fb-TPC-1 and Fb-8505c co-cultures. 
A
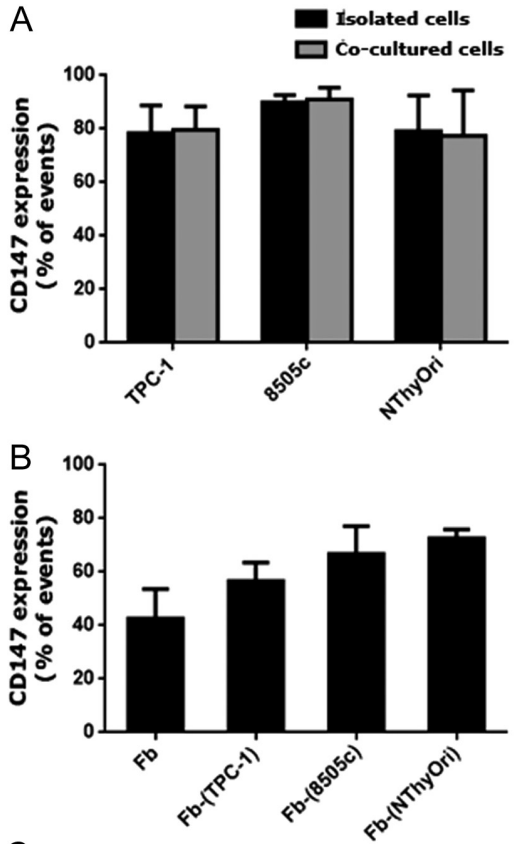

C

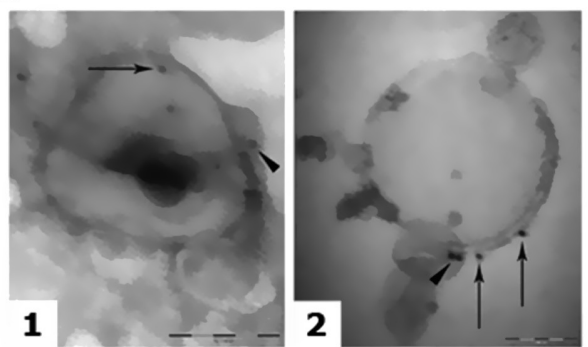

\section{Figure 5}

CD147 expression in Fb, thyroid cells and 100K-EVs from thyroid cells and Fb-thyroid cell co-culture. (A, B) Normal Fb were pre-stained with Cell Proliferation Dye eFluor 670 (Fb-CPD+) and then co-cultured with TPC-1, $8505 \mathrm{c}$ or NThyOri cells, as stated in the Material and Methods section. Isolated and co-cultured cells were collected, fixed, stained with the appropriate primary antiCD147 antibody and analyzed by flow cytometry, with the percentage of CD147 positive events being depicted. (A) CD147 expression was analyzed in TPC-1, 8505c and NThyOri cells and in their respective co-cultures with $\mathrm{Fb}-\mathrm{CPD}+$. No differences in the percentage of CD147 positive cells were observed between isolated and co-cultured thyroid cells. (B) CD147 expression was analyzed in Fb and in their respective co-cultures with TPC-1, 8505c and NThyOri cells (Fb-(TPC-1), $\mathrm{Fb}$-(8505c) and Fb-(NThyOri), respectively). No significant differences in CD147 expression between isolated and co-cultured Fb were observed. Data are presented as the mean value of at least four independent determinations (mean \pm S.E.M.). (C) Representative microphotograph of immunogold-labeling of CD147 protein ( $15 \mathrm{~nm}$ gold particles) in 100K-EVs from Fb-TPC-1 (1) and Fb-8505c (2) co-cultures. CD147 can be detected in small (arrowheads) and large (arrows) EVs. Bars: $200 \mathrm{~nm}$.

\section{CD147 expression increases in EVs obtained from thyroid tumor cell-Fb co-cultures}

To further characterize the released 100K-EVs populations, we tested CD81, a classical tetraspanin marker, typically found in EVs pelleting at high speed (31), and CD147

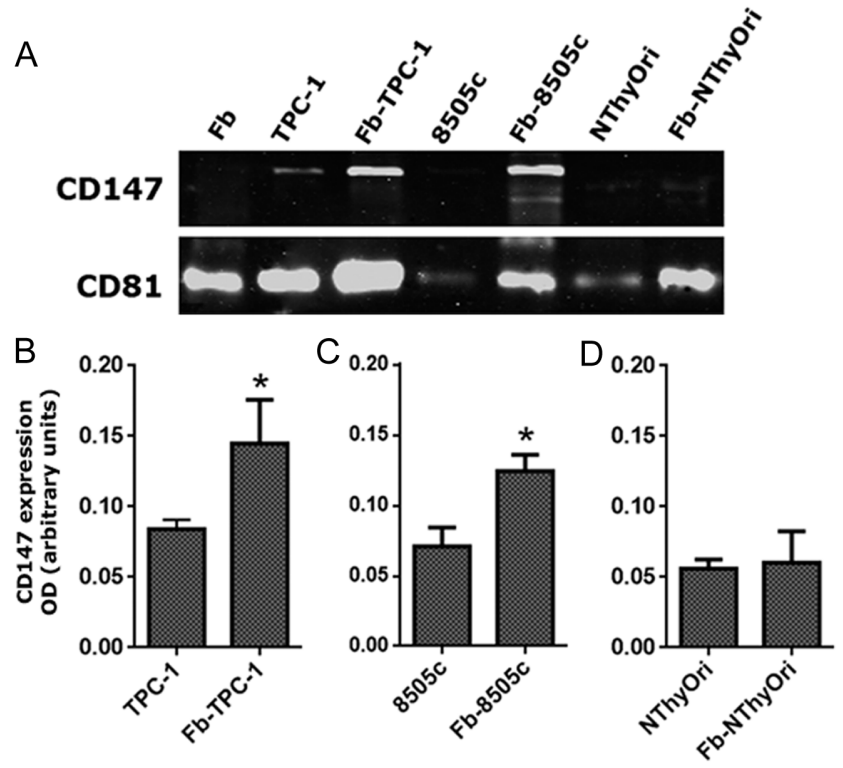

Figure 6

CD147 and CD81 expression in 100K-EVs from isolated and co-cultured thyroid cells. (A) Representative Western blotting assay showing CD147 and CD81 expression in 100K-EVs isolated from Fb, TPC-1, 8505c, NThyOri and Fb-TPC-1, Fb-8505c and Fb-NThyOri co-cultured cells. All EV fractions expressed the classical 100K-EV marker CD81 (A, lower gel). A higher and significant CD147 expression was detected in the 100K-EV fractions obtained from FbTPC-1 and Fb-8505c co-cultures compared with 100K-EV fractions from isolated TPC- 1 and 8505 c cells (A, lower gel; $B, C ; * P<0.05$, Student's $t$-test). No significant differences were detected in CD147 expression in NThyOri and Fb-NThyOri 100K-EVs (D). The results are expressed as mean \pm S.E.M., $n=3$.

expression, by Western blotting in EVs obtained from Fb, TPC-1, 8505c, NThyOri and Fb-TPC-1, Fb-8505c and Fb-NThyOri co-cultures. All EV fractions were found to express the classical EV marker CD81. Furthermore, a significantly higher CD147 expression was detected in 100K-EVs fraction obtained from Fb-TPC-1 and Fb-8505c co-cultures compared to $100 \mathrm{~K}-\mathrm{EV}$ s fractions from isolated TPC-1 and $8505 \mathrm{c}$ cells (Fig. 6A, B and C), but no significant differences were detected in CD147 expression in EVs fractions obtained from NThyOri or Fb-NThyOri co-cultured cells (Fig. 6D).

\section{Discussion}

In the present work, we used the co-culture between tumor cells and normal $\mathrm{Fb}$ as a means of investigating thyroid tumor cell-Fb interaction and its effects on proteolytic activity and thyroid tumor cell migration and also examined the role played by EVs as mediators of thyroid cell-Fb interplay.

This work demonstrated that the interaction of Fb with thyroid tumor cells increased the secretion of 
proMMP2 and proMMP9 to culture supernatants. In turn, a stimulation of MMP2 activity in thyroid tumor cell-Fb co-cultures was observed, which did not take place in non-tumor thyroid cell-Fb co-cultures. Typically, MMP expression is related to tumor aggressiveness and poor patient prognosis in several tumor pathologies, with an increased MMP2 and MMP9 activity in tumor biopsies having been associated with cancer progression in ovarian cancer (31) and in breast cancer tissues $(32,33)$. In addition, the increased expression of MMP2 in tumor and stromal cells was also related to tumor progression and metastasis in prostate cancer (34). In agreement, we also reported a higher and significant proMMP9 activity in human whole prostate cancer tissues compared with proMMP9 activity in benign prostatic hyperplasia (35).

Concerning the MMPs role in thyroid cancer, an increased expression of MMP2 and MMP9 in tumor tissues was positively correlated with lymph node metastasis in PTC patients (36). In agreement, recent studies have described serum MMP2 levels as being a relevant factor in the evaluation of PTC patient prognosis and also preoperative serum MMP2 levels as a predictor of lymph node metastasis and disease persistence/recurrence in male patients (37). Using a cellular model, in the present study, we demonstrated that thyroid tumor cell-Fb interaction provides a permissive milieu for the expression of MMP2 and MMP9 enzymes, thereby demonstrating the role of $\mathrm{Fb}$ in a thyroid tumoral context.

The ability of cancer cells to migrate and invade surrounding tissues and reach distant organs is a crucial hallmark of cancer, with changes in cell cytoskeletal rearrangements, an initial step in the epithelial to mesenchymal transition (EMT) process, being required for cell migration. In our study, there was evidence of a role for tumor-stroma crosstalk in the generation of signals that increase the migratory phenotype of tumor thyroid cells. Related to this, CMs from $\mathrm{Fb}$ or from Fb-thyroid tumor cell co-cultures increased the number of thyroid tumor cells with filopodia and lamellipodia. Furthermore, there was an enhanced cell migration in 8505c cells stimulated with Fb- or Fb-8505c CMs in close association with an increased migratory phenotype. In agreement with our model, Salvatore and coworkers (38) also demonstrated that osteosarcoma malignant cells alter their morphology in human Fb-tumor cell co-cultures, resulting in cytoskeletal polarization and cell protrusions. These authors also described a gradually increasing tumor cell migration during tumor cell co-culture with normal $\mathrm{Fb}$, responding to $\mathrm{Fb}$-secreted paracrine signals.
In addition to their role in matrix degradation, MMPs are strong candidates for initiating both the EMT and the migratory signals that control the initial migration in epithelial cells. In cancer cells, the gelatinases MMP2 and MMP9 are often assumed to be linked to the breakdown of the basement membrane, thereby being involved in the metastasis processes. However, numerous reports have also implicated MMP2 and MMP9 in regulating epithelial cell migration. With respect to this, active MMP2 has been described accelerating human nasal epithelial cell migration (39), with MMP9 overexpression observed to facilitate keratinocyte migration in an in vitro wound assay $(40,41)$ and with both enzymes being critical effectors for invasion in papillary and follicular thyroid cancer cell lines (42). Moreover, in human breast cancer cells, active MMP2 triggers signaling pathways, resulting in actin polymerization and thereby inducing cell migration (43). In another study, in an anaplastic thyroid tumor metastasis model and in thyroid tumor cells, the activation of the TNF receptor-associated factor 6/CD147/ MMP9 pathway induced an increase in MMP9 activity that was positively correlated with tumor metastasis and cell migration (44). Similarly, our results showed a higher migratory phenotype in the anaplastic tumor cell line, which also exerts an enhanced activity of MMP9 and MMP2.

In recent years, many studies have supported the role and importance of EVs in tumor development (45). During tumor progression, an increased EV secretion and specific loading of tumor-promoting factors in tumor-derived EVs have been reported. In the present work, we demonstrated that EV populations were also obtained from isolated and co-cultured thyroid cells, but remarkably, only those vesicles derived from tumor cell-Fb interacting cells were able to modify the proteolytic performance of normal $\mathrm{Fb}$ and clearly acted as vehicles for the delivery of tumor information in the tumor microenvironment. These EVs transport CD147, and interestingly, the expression of CD147 was significantly increased in EV populations obtained from thyroid tumor cell-Fb co-cultures, compared to those from isolated thyroid tumor cells. CD147 has been mainly related to MMPs secretion and activation (46) as well as being linked to tumor-stroma crosstalk (47). In addition, CD147 has been described in EVs isolated from several tumor cell lines and primary tumor-derived cells, with EVs carrying CD147 having been linked to heterotypic cellular interactions. In this sense, ovary cancer cell-derived EVs carrying CD147 have been related to proangiogenic activity in human endothelial cells (8) and the CD147 present in normal

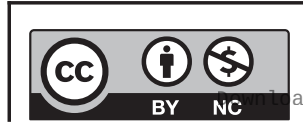

This work is licensed under a Creative Commons Attribution-NonCommercial 4.0 International License. ded from Bioscientifica.com at 04/26/2023 11:07:08AM 


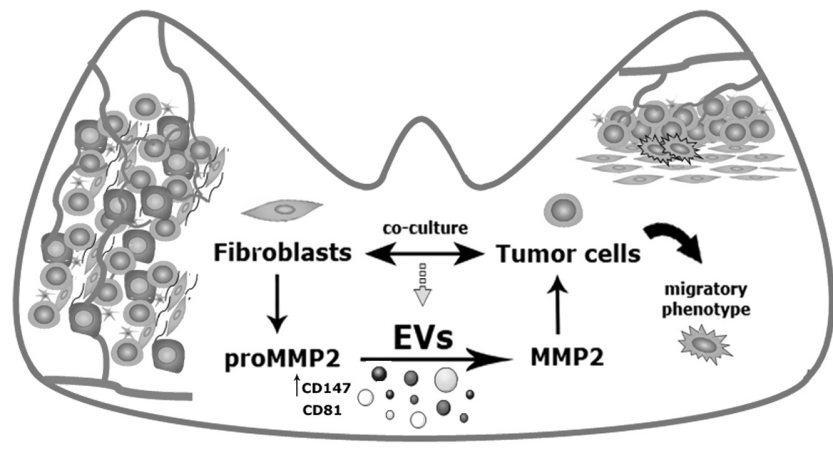

Figure 7

Proposed model describing the role of thyroid tumor cell-Fb interplay in the release of EVs in tumor milieu, leading to metalloproteinases secretion/activation from $\mathrm{Fb}$, favoring a tumor promoting microenvironment in thyroid cancer.

uterine epithelial cell-derived EVs has been linked to MMP induction in normal $\mathrm{Fb}$ (48). In the thyroid gland, CD147 is significantly expressed in thyroid malignant tumor tissues, but not in normal tissues or nodular goiter. Moreover, in differentiated thyroid malignant tumors, CD147 expression is correlated with extrathyroidal invasion and lymph node metastasis and is associated with a lower degree of cellular differentiation $(29,49)$. In line with this, in undifferentiated thyroid carcinomas, a higher expression of CD147 in thyroid tumor cells was observed with respect to differentiated forms of the disease (50).

Although our in vitro model is a simplified version of the thyroid tumor microenvironment, where 2D-interactions are displayed and only two different cells participate of these interactions, we were able to show for the first time the relevance of tumor-stroma interplay in the release of a qualified different population of EVs in thyroid cancer. Besides, even though other limitation of our study resides in that the intimate mechanism of EVs interaction with $\mathrm{Fb}$ as well as the finely participation of EVs in MMPs activation are still unknown, the present work introduces CD147 expression in another thyroid tumoral context, that of being a molecule with the ability to be detected in EVs, and thus opening the possibility of its detection in accessible bodily fluids. This finding opens new frontiers of study with putative impact on human oncology, which is the goal of ongoing investigation.

To conclude, our experimental design, using a simulation of the thyroid tumor microenvironment, suggests a possible role for thyroid tumor cell-Fb interaction in the promotion of the tumor cell migratory phenotype as well as MMP2 and MMP9 expression. As depicted in Fig. 7, our results suggest the role of $\mathrm{Fb}$ as protagonists of thyroid tumor progression, in consonance with our previous findings (21). As well, they highlight for the first time the role of tumor cell-Fb interaction in EV secretion and also EVs as mediators of tumor-stroma crosstalk in the thyroid tumor milieu, thereby pointing to $\mathrm{Fb}$ as a possible matrix metalloproteinase source in this tumor microenvironment.

\section{Supplementary materials}

This is linked to the online version of the paper at https://doi.org/10.1530/ EC-20-0113.

\section{Declaration of interest}

Claudia G Pellizas is a Senior Editor for Endocrine Connections. She was not involved in the editorial or peer review process of this manuscript, on which she is listed as an author.

\section{Funding}

This work was supported by the Agencia Nacional de Promoción Científica y Tecnológica, Fondo Nacional de Ciencia y Tecnología (AGENCIA-FONCyT; PICT-2016-0254), Secretaría de Ciencia y Tecnología de la Universidad Nacional de Córdoba (SECyT-UNC; 33620180100476CB) and Fundación Sales. Institutional funding from CONICET (Res. 1691/16) is kindly acknowledged.

\section{Acknowledgements}

The authors thank Dr Carolina Leimgruber and PhD student Ezequiel Grondona (INICSA-CONICET) for their assistance in TEM studies and Dr María Cecilia Ramello (CIBICl-CONICET) for her assistance in flow citometry analysis. The authors thank M P Abadie, M P Crespo, A Romero, $L$ Gatica and G Furlán for their excellent technical assistance. The authors thank Dr Eva V Acosta Rodriguez and Dr Anahí Franchi for providing reagents. $R C B M$ is a research fellow of CONICET. A B D V was a research fellow of the National Cancer Institute in Argentina and of CONICET. M L G had a research scholarship from Consejo Interuniversitario Nacional (CIN). A LD P, C G P and A C D are all established researchers at CONICET.

\section{References}

1 Egeblad M, Nakasone ES \& Werb Z. Tumors as organs: complex tissues that interface with the entire organism. Developmental Cell 201018 884-901. (https://doi.org/10.1016/j.devcel.2010.05.012)

2 Spill F, Reynolds DS, Kamm RD \& Zaman MH. Impact of the physical microenvironment on tumor progression and metastasis. Current Opinion in Biotechnology 201640 41-48. (https://doi.org/10.1016/j. copbio.2016.02.007)

3 Kalluri R. The biology and function of fibroblasts in cancer. Nature Reviews: Cancer 201616 582-598. (https://doi.org/10.1038/ nrc.2016.73)

4 Kessenbrock K, Plaks V \& Werb Z. Matrix metalloproteinases: regulators of the tumor microenvironment. Cell 2010141 52-67. (https://doi.org/10.1016/j.cell.2010.03.015)

5 Shay G, Lynch CC \& Fingleton B. Moving targets: emerging roles for MMPs in cancer progression and metastasis. Matrix Biology 2015 44-46 200-206. (https://doi.org/10.1016/j.matbio.2015.01.019)
This work is licensed under a Creative Commons Attribution-NonCommercial 4.0 International License. ded from Bioscientifica.com at 04/26/2023 11:07:08AM 
6 Grass GD \& Toole BP. How, with whom and when: an overview of CD147-mediated regulatory networks influencing matrix metalloproteinase activity. Bioscience Reports 201536 e00283. (https://doi.org/10.1042/BSR20150256)

7 Xiong L, Edwards 3rd CK \& Zhou L. The biological function and clinical utilization of CD147 in human diseases: a review of the current scientific literature. International Journal of Molecular Sciences 201415 17411-17441. (https://doi.org/10.3390/ijms151017411)

8 Millimaggi D, Mari M, D’Ascenzo S, Carosa E, Jannini EA, Zucker S, Carta G, Pavan A \& Dolo V. Tumor vesicle-associated CD147 modulates the angiogenic capability of endothelial cells. Neoplasia 20079 349-357. (https://doi.org/10.1593/neo.07133)

9 Hatanaka M, Higashi Y, Fukushige T, Baba N, Kawai K, Hashiguchi T, Su J, Zeng W, Chen X \& Kanekura T. Cleaved CD147 shed from the surface of malignant melanoma cells activates MMP2 produced by fibroblasts. Anticancer Research 201434 7091-7096.

10 Donadio AC, Remedi MM, Susperreguy S, Frede S, Gilardoni MB, Tang Y, Pellizas CG \& Yan L. Extracellular matrix metalloproteinase inducer (EMMPRIN) and matrix metalloproteinases (MMPs) as regulators of tumor-host interaction in a spontaneous metastasis model in rats. Histochemistry and Cell Biology 2008130 1155-1164. (https://doi.org/10.1007/s00418-008-0496-6)

11 Tkach M \& Thery C. Communication by extracellular vesicles: where we are and where we need to go. Cell 2016164 1226-1232. (https:// doi.org/10.1016/j.cell.2016.01.043)

12 Maas SLN, Breakefield XO \& Weaver AM. Extracellular vesicles: unique intercellular delivery vehicles. Trends in Cell Biology 201727 172-188. (https://doi.org/10.1016/j.tcb.2016.11.003)

13 Wendler F, Favicchio R, Simon T, Alifrangis C, Stebbing J \& Giamas G. Extracellular vesicles swarm the cancer microenvironment: from tumor-stroma communication to drug intervention. Oncogene 201736 877-884. (https://doi.org/10.1038/ onc.2016.253)

14 Willms E, Cabanas C, Mager I, Wood MJA \& Vader P. Extracellular vesicle heterogeneity: subpopulations, isolation techniques, and diverse functions in cancer progression. Frontiers in Immunology 2018 9 738. (https://doi.org/10.3389/fimmu.2018.00738)

15 Arendt BK, Walters DK, Wu X, Tschumper RC \& Jelinek DF. Multiple myeloma dell-derived microvesicles are enriched in CD147 expression and enhance tumor cell proliferation. Oncotarget 20145 5686-5699. (https://doi.org/10.18632/oncotarget.2159)

16 Menck K, Scharf C, Bleckmann A, Dyck L, Rost U, Wenzel D, Dhople VM, Siam L, Pukrop T, Binder C, et al. Tumor-derived microvesicles mediate human breast cancer invasion through differentially glycosylated EMMPRIN. Journal of Molecular Cell Biology 20157 143-153. (https://doi.org/10.1093/jmcb/mju047)

17 Willms A, Muller C, Julich H, Klein N, Schwab R, Gusgen C, Richardsen I, Schaaf S, Krawczyk M, Krawczyk M, et al. Tumourassociated circulating microparticles: a novel liquid biopsy tool for screening and therapy monitoring of colorectal carcinoma and other epithelial neoplasia. Oncotarget 20167 30867-30875. (https://doi. org/10.18632/oncotarget.9018)

18 Siegel RL, Miller KD \& Jemal A. Cancer statistics, 2019. CA: A Cancer Journal for Clinicians 201969 7-34. (https://doi.org/10.3322/ caac.21551)

19 Jolly LA, Novitskiy S, Owens P, Massoll N, Cheng N, Fang W, Moses HL \& Franco AT. Fibroblast-mediated collagen remodeling within the tumor microenvironment facilitates progression of thyroid cancers driven by BrafV600E and Pten loss. Cancer Research 201676 1804-1813. (https://doi.org/10.1158/0008-5472.CAN-15-2351)

20 Saitoh O, Mitsutake N, Nakayama T \& Nagayama Y. Fibroblastmediated in vivo and in vitro growth promotion of tumorigenic rat thyroid carcinoma cells but not normal Fisher rat thyroid follicular cells. Thyroid 200919 735-742. (https://doi.org/10.1089/ thy.2009.0017)
21 Fozzatti L, Alamino VA, Park S, Giusiano L, Volpini X, Zhao L, Stempin CC, Donadio AC, Cheng SY \& Pellizas CG. Interplay of fibroblasts with anaplastic tumor cells promotes follicular thyroid cancer progression. Scientific Reports 20199 8028. (https://doi. org/10.1038/s41598-019-44361-6)

22 Martinez-Outschoorn UE, Pavlides S, Whitaker-Menezes D, Daumer KM, Milliman JN, Chiavarina B, Migneco G, Witkiewicz AK, Martinez-Cantarin MP, Flomenberg N, et al. Tumor cells induce the cancer associated fibroblast phenotype via caveolin-1 degradation: implications for breast cancer and DCIS therapy with autophagy inhibitors. Cell Cycle 20109 2423-2433. (https://doi.org/10.4161/ cc.9.12.12048)

23 Desrochers LM, Bordeleau F, Reinhart-King CA, Cerione RA \& Antonyak MA. Microvesicles provide a mechanism for intercellular communication by embryonic stem cells during embryo implantation. Nature Communications 20167 11958. (https://doi. org/10.1038/ncomms11958)

24 Tkach M, Kowal J \& Thery C. Why the need and how to approach the functional diversity of extracellular vesicles. Philosophical Transactions of the Royal Society of London: Series B, Biological Sciences 2018373 20160479. (https://doi.org/10.1098/rstb.2016.0479)

25 De Paul AL, Attademo AM, Caron RW, Soaje M, Torres AI, Jahn GA \& Celis ME. Neuropeptide glutamic-isoleucine (NEI) specifically stimulates the secretory activity of gonadotrophs in primary cultures of female rat pituitary cells. Peptides 200930 2081-2087. (https://doi. org/10.1016/j.peptides.2009.08.022)

26 Le Clainche C \& Carlier MF. Regulation of actin assembly associated with protrusion and adhesion in cell migration. Physiological Reviews 200888 489-513. (https://doi.org/10.1152/physrev.00021.2007)

27 Shimoda M \& Khokha R. Metalloproteinases in extracellular vesicles. Biochimica et Biophysica Acta: Molecular Cell Research 20171864 1989-2000. (https://doi.org/10.1016/j.bbamcr.2017.05.027)

28 Becker A, Thakur BK, Weiss JM, Kim HS, Peinado H \& Lyden D. Extracellular vesicles in cancer: cell-to-cell mediators of metastasis. Cancer Cell 201630 836-848. (https://doi.org/10.1016/j. ccell.2016.10.009)

29 Huang P, Mao LF, Zhang ZP, Lv WW, Feng XP, Liao HJ, Dong C, Kaluba B, Tang XF \& Chang S. Down-regulated miR-125a-5p promotes the reprogramming of glucose metabolism and cell malignancy by increasing levels of CD147 in thyroid cancer. Thyroid 201828 613-623. (https://doi.org/10.1089/thy.2017.0401)

30 Sidhu SS, Mengistab AT, Tauscher AN, LaVail J \& Basbaum C. The microvesicle as a vehicle for EMMPRIN in tumor-stromal interactions. Oncogene 200423 956-963. (https://doi.org/10.1038/ sj.onc. 1207070

31 Schmalfeldt B, Prechtel D, Harting K, Spathe K, Rutke S, Konik E, Fridman R, Berger U, Schmitt M, Kuhn W, et al. Increased expression of matrix metalloproteinases (MMP)-2, MMP-9, and the urokinasetype plasminogen activator is associated with progression from benign to advanced ovarian cancer. Clinical Cancer Research 20017 2396-2404.

$32 \mathrm{Li} \mathrm{H}$, Qiu Z, Li F \& Wang C. The relationship between MMP-2 and MMP-9 expression levels with breast cancer incidence and prognosis. Oncology Letters 201714 5865-5870. (https://doi.org/10.3892/ ol.2017.6924)

33 Mehner C, Hockla A, Miller E, Ran S, Radisky DC \& Radisky ES. Tumor cell-produced matrix metalloproteinase 9 (MMP-9) drives malignant progression and metastasis of basal-like triple negative breast cancer. Oncotarget 20145 2736-2749. (https://doi. org/10.18632/oncotarget.1932)

34 Murray NP, Reyes E, Tapia P, Badinez L \& Orellana N. Differential expression of matrix metalloproteinase- 2 expression in disseminated tumor cells and micrometastasis in bone marrow of patients with nonmetastatic and metastatic prostate cancer: theoretical considerations and clinical implications-an immunocytochemical https://ec.bioscientifica.com https://doi.org/10.1530/EC-20-0113

C) 2020 The authors Published by Bioscientifica Ltd
This work is licensed under a Creative Commons Attribution-NonCommercial 4.0 International License. ed from Bioscientifica, com at 04/26/2023 11:07:08AM 
study. Bone Marrow Research 20122012 259351. (https://doi. org/10.1155/2012/259351)

35 Gilardoni MB, Remedi MM, Oviedo M, Dellavedova T, Sarria JP, Racca L, Dominguez M, Pellizas CG \& Donadio AC. Differential expression of low density lipoprotein receptor-related protein 1 (LRP-1) and matrix metalloproteinase-9 (MMP-9) in prostate gland: from normal to malignant lesions. Pathology: Research and Practice 2017213 66-71. (https://doi.org/10.1016/j.prp.2016.11.008)

36 Huang LL, Wang Z, Cao CJ, Ke ZF, Wang F, Wang R, Luo CQ, Lu X \& Wang LT. AEG-1 associates with metastasis in papillary thyroid cancer through upregulation of MMP2/9. International Journal of Oncology 201751 812-822. (https://doi.org/10.3892/ijo.2017.4074)

37 Shi Y, Su C, Hu H, Yan H, Li W, Chen G, Xu D, Du X \& Zhang P. Serum MMP-2 as a potential predictive marker for papillary thyroid carcinoma. PLoS ONE 201813 e0198896. (https://doi.org/10.1371/ journal.pone.0198896)

38 Salvatore V, Teti G, Focaroli S, Mazzotti MC, Mazzotti A \& Falconi M. The tumor microenvironment promotes cancer progression and cell migration. Oncotarget 20178 9608-9616. (https://doi.org/10.18632/ oncotarget.14155)

39 Lechapt-Zalcman E, Pruliere-Escabasse V, Advenier D, Galiacy S, Charriere-Bertrand C, Coste A, Harf A, d'Ortho MP \& Escudier E. Transforming growth factor-beta1 increases airway wound repair via MMP-2 upregulation: a new pathway for epithelial wound repair? American Journal of Physiology: Lung Cellular and Molecular Physiology 2006290 L1277-L1282. (https://doi.org/10.1152/ajplung.00149.2005)

40 Seomun Y, Kim JT \& Joo CK. MMP-14 mediated MMP-9 expression is involved in TGF-beta1-induced keratinocyte migration. Journal of Cellular Biochemistry 2008104 934-941. (https://doi.org/10.1002/ jcb.21675)

41 Kyriakides TR, Wulsin D, Skokos EA, Fleckman P, Pirrone A, Shipley JM, Senior RM \& Bornstein P. Mice that lack matrix metalloproteinase-9 display delayed wound healing associated with delayed reepithelization and disordered collagen fibrillogenesis. Matrix Biology 200928 65-73. (https://doi.org/10.1016/j. matbio.2009.01.001)

42 Yeh MW, Rougier JP, Park JW, Duh QY, Wong M, Werb Z \& Clark OH. Differentiated thyroid cancer cell invasion is regulated through epidermal growth factor receptor-dependent activation of matrix metalloproteinase (MMP)-2/gelatinase A. Endocrine-Related Cancer 200613 1173-1183. (https://doi.org/10.1677/erc.1.01226)

43 Das K, Prasad R, Ansari SA, Roy A, Mukherjee A \& Sen P. Matrix metalloproteinase-2: a key regulator in coagulation proteases mediated human breast cancer progression through autocrine signaling. Biomedicine and Pharmacotherapy 2018105 395-406. (https://doi.org/10.1016/j.biopha.2018.05.155)

44 Shi GH \& Zhou L. Emodin suppresses angiogenesis and metastasis in anaplastic thyroid cancer by affecting TRAF6mediated pathways in vivo and in vitro. Molecular Medicine Reports 201818 5191-5197. (https://doi.org/10.3892/mmr.2018.9510)

45 Xu R, Rai A, Chen M, Suwakulsiri W, Greening DW \& Simpson RJ. Extracellular vesicles in cancer - implications for future improvements in cancer care. Nature Reviews: Clinical Oncology 2018 15 617-638. (https://doi.org/10.1038/s41571-018-0036-9)

46 Tang W, Chang SB \& Hemler ME. Links between CD147 function, glycosylation, and caveolin-1. Molecular Biology of the Cell 200415 4043-4050. (https://doi.org/10.1091/mbc.e04-05-0402)

47 Tang Y, Kesavan P, Nakada MT \& Yan L. Tumor-stroma interaction: positive feedback regulation of extracellular matrix metalloproteinase inducer (EMMPRIN) expression and matrix metalloproteinasedependent generation of soluble EMMPRIN. Molecular Cancer Research 20042 73-80.

48 Braundmeier AG, Dayger CA, Mehrotra P, Belton Jr RJ \& Nowak RA. EMMPRIN is secreted by human uterine epithelial cells in microvesicles and stimulates metalloproteinase production by human uterine fibroblast cells. Reproductive Sciences 201219 1292-1301. (https://doi.org/10.1177/1933719112450332)

49 Tan H, Ye K, Wang Z \& Tang H. CD147 expression as a significant prognostic factor in differentiated thyroid carcinoma. Translational Research 2008152 143-149. (https://doi.org/10.1016/j. trsl.2008.07.005)

50 Huang P, Chang S, Jiang X, Su J, Dong C, Liu X, Yuan Z, Zhang Z \& Liao H. RNA interference targeting CD147 inhibits the proliferation, invasiveness, and metastatic activity of thyroid carcinoma cells by down-regulating glycolysis. International Journal of Clinical and Experimental Pathology 20158 309-318.

Received in final form 11 April 2020

Accepted 12 May 2020

Accepted Manuscript published online 12 May 2020
This work is licensed under a Creative Commons Attribution-NonCommercial 4.0 International License. ded from Bioscientifica.com at 04/26/2023 11:07:08AM 